\title{
Solar and Interplanetary Particles Re-accelerated at the Solar Wind Termination Shock
}

\author{
R. A. Mewaldt \\ California Institute of Technology, Pasadena, CA 91125
}

\begin{abstract}
We consider the acceleration of solar/interplanetary particles at the solar wind termination shock, predict the expected composition and energy spectra, and discuss possible observable consequences.
\end{abstract}

\section{Introduction}

The "anomalous" cosmic rays (ACRs), which include the elements $\mathrm{H}, \mathrm{He}, \mathrm{C}$, $\mathrm{N}, \mathrm{O}, \mathrm{Ne}$, and $\mathrm{Ar}$, originate from interstellar neutral particles that have been swept into the heliosphere, ionized by solar UV or charge exchange with the solar wind, convected into the outer heliosphere, and then accelerated to -5 to $50 \mathrm{MeV} /$ nuc [1]. It is commonly assumed that the bulk of ACR acceleration occurs at the solar wind termination shock [2] by diffusive shock acceleration [3], although some pre-acceleration may occur in interplanetary space [4].

Jokipii [5] pointed out that the termination shock would also be expected to accelerate (or re-accelerate) other species, including solar and interplanetary ions, and Jovian electrons. This paper considers the re-acceleration of lowenergy solar/interplanetary particles at the termination shock, and identifies possible observable consequences. The seed population for these "reaccelerated solar energetic particles" (RSEPs), might include solar energetic particles (SEPs), interplanetary ions energized by corotating-interaction regions (CIRs) and other shocks, as well as the solar wind (SW).

\section{Composition, Charge States, and Properties of RSEPs}

The composition and energy spectra of ACRs are known to scale in a simple fashion with the mass to charge ratio (AVQ) [6]. We extend this relation to predict the expected properties of RSEPs by taking into account the relative $\mathrm{A} / \mathrm{Q}$ ratios and abundances of solar particles. Ignoring minor differences between the abundances of SEPs, CIR-associated ions, and SW ions, we adopt the average SEP elemental abundances in Stone [7], assume $\mathrm{H} / \mathrm{He}=20$, and for SEP charge-states use the measurements of Luhn et al. [10].

Cummings, Stone, and Webber ([6]; CSW) showed that the observed spectra of ACR $\mathrm{He}, \mathrm{N}, \mathrm{O}$, and $\mathrm{Ne}$ all have a generic spectral shape whose characteristic energy scales with the AVQ ratio. For the observed 1977-78 spectra at $\sim 2 \mathrm{AU}$ the peaks occurred at an energy given by $\mathrm{E}_{\mathrm{p}}=53(\mathrm{~A} / \mathrm{Q})^{-0.91}$, where $\mathrm{Q}=1$ for $\mathrm{ACR}$. This generic shape evolves over the solar cycle, and in a later Voyager study at $23 \mathrm{AU}$ in the $\mathrm{A}<0$ portion of the 22-year solar cycle [9] the peaks were shifted such that $E_{p}=145(A / Q)^{-1.29}$. Assuming that these scaling relations also apply to other ions accelerated at the termination shock, Figure 1 shows the predicted locations of the peak flux of RSEP ions for these two periods. Based on observations of ACR He [10] we would expect the 1987 spectra at $1 \mathrm{AU}$ to peak at energies $\sim 25 \%$ higher than at $23 \mathrm{AU}$. Although the 
peaks of the ACR spectra at 1 AU during 1992-1995 are not yet well established, they should be similar to those observed 22 years earlier.

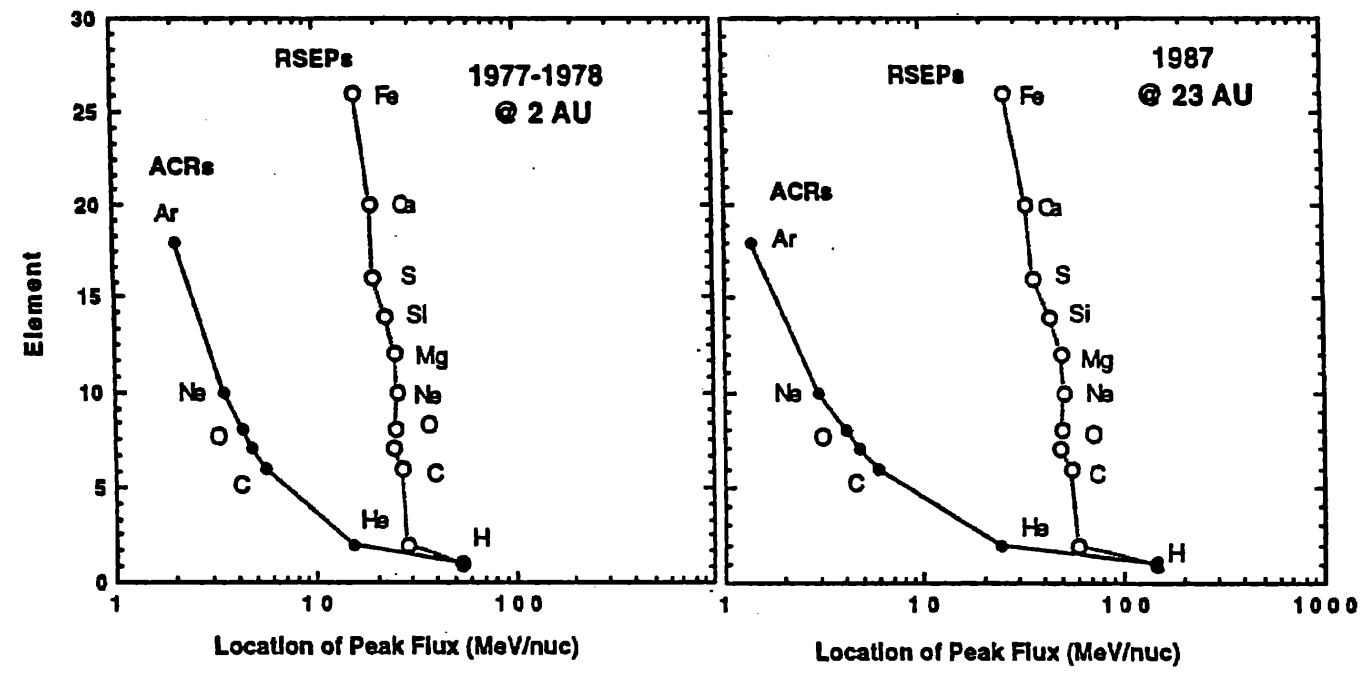

Figure 1: Predicted location of the peak flux of "anomalous" solar particles, scaled from the observed locations of the ACR peaks, for 1977-78 and 1987.

CSW [6] also found that the relative intensity of the various species (measured at the point of peak intensity) scaled with $\mathrm{A} / \mathrm{Q}$, thereby relating the observed ACR abundances to the source composition of pickup ions. To quantify this relation, we assume a $\mathrm{He} / \mathrm{Ne}$ ratio of 300 for interstellar neutrals. The 1977-1978 ACR intensities are then given by $\mathrm{J}_{\mathrm{i}} \sim \mathrm{A}_{\mathrm{i}}(\mathrm{A} / \mathrm{Q})^{1.29}$, with $\mathrm{J}_{\mathrm{i}} \sim$ $\mathrm{A}_{\mathrm{i}}(\mathrm{A} / \mathrm{Q})^{2.0}$ for 1987 at $23 \mathrm{AU}$. Here $\mathrm{A}_{\mathrm{i}}$ is the calculated pickup ion abundance for species $i$ [11]. Note, however, that this intensity scaling may not apply to RSEPs if the dependence of the injection efficiencies for pickup ions and RSEPs differ in their dependence on A/Q.

To estimate whether RSEPs would be observable, it is necessary to relate their expected fluxes to those of ACRs and galactic cosmic rays (GCRs). Although SEP \& CIR ions are much less abundant than pickup ions in the outer heliosphere, they should be more efficiently accelerated to high energies, since they are injected at much higher energies (e.g., 0.1 to $1 \mathrm{MeV} /$ nuc vs. $\sim 1$ $\mathrm{keV} / \mathrm{nuc}$ for pickup ions). For illustration, Figure 2 shows representative spectra of RSEPs, ACRs, and GCRs that follow from assuming that the peak flux of RSEP oxygen is $0.25 \%$ of that of ACR oxygen. Since the RSEP contributions for species such as $\mathrm{He}, \mathrm{N}, \mathrm{O}$, and $\mathrm{Ne}$ are likely to be buried under the ACR contributions, it appears that RSEP contributions are most likely to be detectable in heavy species such as Si and Fe.

\section{Consequences and Possible Evidence for RSEPs}

There are several key properties that might lead to the identification of RSEPs. The most obvious signature would be low energy enhancements in the quiet-time spectra of species such as $\mathrm{Mg}, \mathrm{Si}$, and $\mathrm{Fe}$ that are not expected to have ACR contributions. Recently, an excess of $\sim 10$ to $15 \mathrm{MeV} /$ nuc carbon has been reported well above that expected from ACRs $([12,13]$, at an energy 
consistent with that expected for RSEPs. If this C feature is due to RSEPs, one would also expect features in other species such as $\mathrm{Si}$ and $\mathrm{Fe}$, that should be searched for. There have also been previous reports of low-energy spectral features [14]. Note, however, that such features might also be of solar or interplanetary origin. Short of producing a clear "bump", RSEPs might simply lead to a "flattening" of the typical J=aT form of low-energy GCR spectra. While there have been reports of low-energy GCR spectra differing from J=aT (e.g.[15]), such spectra might also result from reduced solar modulation.

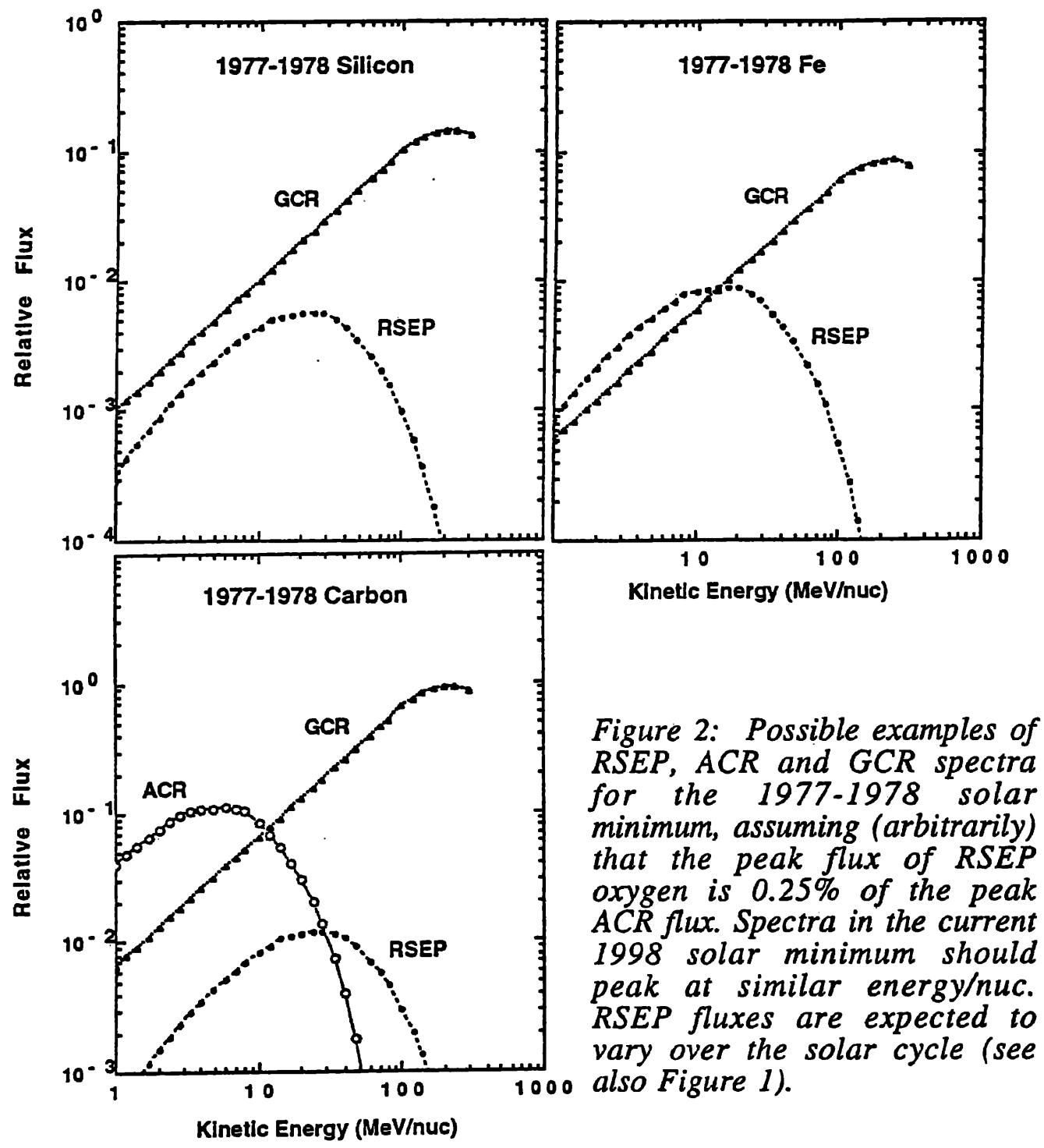

RSEP contributions might also be reflected in "secondary/primary" ratios such as $B / C$, or $Z=(21-25) / F e$, since "secondary" species such as $B$ and $Z=21-25$ nuclei are many times more abundant in GCRS than in SEPs. Ferrando et al. [16] reported a reduced abundance of Fe secondaries among GCRs at $23 \mathrm{AU}$ in 1987. While they ascribed this to reduced solar modulation, it could also include the effects of RSEPs. 
Because ACRs are strongly affected by solar modulation, they are observable at $1 \mathrm{AU}$ only at solar minimum (e.g., [12]). The intensity of RSEPs relative to ACRs should be highest near or just after solar maximum, when the abundance of SEP seed nuclei in the outer heliosphere is greatest.

Perhaps the most direct signature of RSEPs would be the observation of partially-stripped $(\mathrm{Q}<\mathrm{Z})$ cosmic rays with energies of $\sim 10$ to $100 \mathrm{MeV} /$ nuc during solar/ quiet periods. Biswas et al. [16] have reported Spacelab-3 (SL-3) observations in low-earth orbit of heavy nuclei with $21 \leq Z \leq 29$ that were apparently partially stripped, since they were observed at geomagnetic latitudes inaccessible to fully-stripped cosmic rays. Similar observations have been reported from S81-1 and CRRES [18]. The SL-3 particles have the expected characteristics of RSEPs, but include a much greater percentage of $21 \leq Z \leq 24$ nuclei than expected from a source with solar composition. Note however, that they also include a large excess of $27 \leq Z$ $\leq 29$ nuclei [19], suggesting that limited charge resolution might account for the $21 \leq \mathrm{Z} \leq 24$ excess. The S81-1 and CRRES compositions do appear consistent with a solar composition. All of these observations occurred following a year of major solar activity. They could represent RSEPs, and these data deserve further study.

Finally, if RSEPs are indeed accelerated at the termination shock, Voyager and Pioneer- 10 may observe spectral features in species such as $\mathrm{Si}$ and $\mathrm{Fe}$ as precursors of their approach to the termination shock.

In conclusion, while there is presently no "smoking gun" proving the existence of RSEPs, there are several possible clues, and it appears worthwhile to search further for their presence in both the outer and inner heliosphere.

Acknowledgments: I appreciate conversations with J. R. Jokipii, who first suggested to me that SEPs might be further accelerated at the termination

shock. I also thank A. C. Cummings and E. C. Stone. This work was supported by NASA under NAS5-30704 \& NAGW-1919.

\section{References}

[1] Fisk, L.A., B. Kozlovsky \& R. Ramaty, ApJ 190(1974)L35

[2] Pesses, M.E., J.R. Jokipii and D. Eichler, ApJ 246(1981)L85

[3] Jokipii, J.R., JGR 91(1986)2929

[4] Gloeckler, G., et al., JGR 99(1994)17637

[5] Jokipii, J.R., private communication, December 1991.

[6] Cummings, A. C., Stone, E. C., and Webber, W. R., ApJ 287(1984)L99

[7] Stone, E.C., in AIP Conf. Proceedings 183(1989)100

[8] Luhn, A., et al., Proc. 19th ICRC (La Jolla), 4(1985)241

[9] Cummings, A.C. \& E.C. Stone, Proc. 21st ICRC (Adelaide) 6(1990)202

[10] McDonald, F.B., et al., JGR 97(1992)1557

[11] Cummings, A.C., private communication, July 1994.

[12] Mewaldt, R.A., et al., Geo. Res., Lett. 20(1993)2263

[13] Hasebe, N., et al., GRL 21(1994)3027

[14] Webber, W.R., E. C. Stone, \& R.E. Vogt, Proc. 16th ICRC 5(1979)357

[15] Garcia-Munoz, M., et al., Proc. 15th ICRC (Plovdiv) 1(1977)230

[16] Ferrando P., et al., Proc. 21st ICRC (Adelaide) 6(1990)202

[17] Biswas, S., et al., ApJ 359(1990)L5; Dutta, A. et al. ApJ 411(1993)418

[18] Cooper, J.F., et al., Adv. Space Res. 15(1995), p. (1)61.

[19] Biswas , S. et al., Preprint TIFR-CRSP-94-1 